\title{
Noise Removal in Magnetic Resonance Images using Hybrid KSL Filtering Technique
}

\author{
C. Lakshmi Devasena \\ Department of Software Systems \\ Karpagam University \\ Coimbatore-21
}

\author{
M. Hemalatha \\ Department of Software Systems \\ Karpagam University \\ Coimbatore-21
}

\begin{abstract}
In Medical Diagnostic, Magnetic Resonance Images play a major role. Magnetic Resonance images are normally corrupted by random noise from the measurement process complicating the automatic feature extraction and analysis of clinical data. Because of this reason noise removal methods have been customarily applied to improve MR image quality. This work proposed a new scheme based on applying a series of filters, each used to modify the estimate into greater agreement, so that the output converges to a stable estimate providing noise free image. In this work, we have introduced a novel hybrid filter to reduce random noise in MR images by the combination of Kernel, Sobel and Low-pass (KSL) filtering techniques. The proposed method has been implemented using Matlab and compared with related state of art methods over synthetic and real clinical MR images showing a superior performance in all cases analyzed.
\end{abstract}

General Terms: Noise Removal - Magnetic Resonance Images.

Keywords: Kernel Operator, Low-pass Filter, Noise Removal, Sobel Operator.

\section{INTRODUCTION}

Noise removal is the process of removing noise from a signal. Noise reduction techniques are theoretically comparable regardless of the signal being processed. Image denoising is often used in the field of publishing or photography where an image was degraded somehow but needs to be enhanced before it can be printed. Denoising is playing an important role in Medical image enhancement also.

Retrieving a high quality MR Image for a medical diagnostic is critical, because it injures human more if we pass high level Magnetic resonance sound to take the image. So denoising of magnetic resonance (MR) images is a challenging issue. MR images are normally corrupted by thermal noise, sample resolution, etc. Understanding the spatial distribution of noise in an MR image is very difficult to any attempt to calculate approximately the true signal. The investigation of how noise is distributed in MR images is chronological.

\section{RELATED WORKS}

Various algorithms used for image denoising are discussed in [1]. The de-noising of Magnetic Resonance Images using wave atom shrinkage is proposed in [2] and also proved that this approach achieves a better SNR compared to wavelet and curvelet shrinkages. A NL-Denoising method for Rician noise reduction is proposed in [3 \& 4]. In [5], a test bed for baseline correction and noise filtering methods is implemented and compared. In [6] a nonparametric Neighborhood Statistics method is proposed for MRI Denoising. An adaptive waveletbased Magnetic Resonance images denoising algorithm using wavelet shrinkage and mixture model concept is introduced in [7]. The method to improve image quality based on determining the critical pulse sequence parameters by timing constraints from all gradients, rather than a single gradient of the image has been given in [8]. A new filter to reduce random noise in multicomponent MR images by spatially averaging similar pixels and a local principal component analysis decomposition using information from all available image components to perform the denoising process is proposed in [9]. A new signal estimator based upon the technique of "noise cancellation", which is commonly used in signal processing is used to recover signals corrupted by additive noise in MRI is proposed in [10]. An estimator using a priori information for devising a single dimensional noise cancellation for the variance of the thermal noise in magnetic resonance imaging (MRI) systems called ML estimator has been proposed in [11]. Non-Local Means (NLM) filtering method for reducing artifacts caused in MRI due to under sampling of $\mathrm{k}$-space (to reduce scan time) is proposed in [12]. A maximum a posteriori estimation technique that operates directly on the diffusion weighted images and accounts for the biases introduced by Rician noise is introduced in [13] for filtering diffusion tensor magnetic resonance images. A novel approach to evaluating reconstructions for low-SNR magnetic resonance (MR) images is given in [14]. A filtering process based on anisotropic diffusion is presented in [15]. A spatially adaptive TV model has been applied to partially parallel MRI (PP-MRI) image reconstructed using GRAPPA ( generalized approach to parallel magnetic resonance imaging) and SENSE (Sensitivity encoding MRI Imaging) is proposed in [16]. The novel filtering method known as trilateral filtering (TF) is proposed in [17 and 18] works similar as Bilateral Filtering and takes the geometric, photometric and local structural similarities to smooth the MR images. A noise removal technique using 4th order PDE is introduced in [19] to reduce noise in MRI images. A phase error estimation scheme based on iteratively applying a series of non-linear filters each used to modify the estimate into greater agreement with one piece of knowledge, until the output converges to a stable estimate is introduced in [20]. A waveletbased multiscale products thresholding scheme using Dyadic Wavelet Transform for detecting Multiscale Edge is introduced in [21] for noise suppression of magnetic resonance images. Our proposed work introduces a hybrid filtering technique combining sobel, kernel and low-pass filters to produce a noisereduced MRI image. 


\section{METHODOLOGY AND DESIGN}

There is lots of image processing and statistical and machine learning techniques involved in the design of the proposed system.

\subsection{Proposed Model of the Noise Reduction System}

Fig 1 shows the proposed model of the Noise Reduction System.

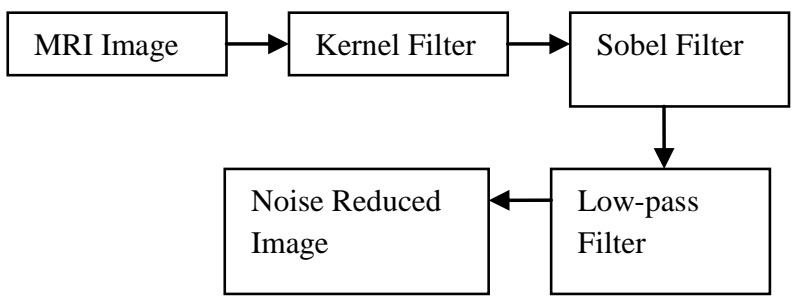

Fig 1: Model of the Noise Removal system

\subsection{Techniques used in Proposed Model}

\subsubsection{Sobel Filter}

The Sobel operator performs a 2-D spatial gradient measurement on an image. Typically it is used to find the approximate absolute gradient magnitude at each point in an input grayscale image. The Sobel edge detector uses a pair of $3 \times 3$ convolution masks, one estimating the gradient in the $\mathrm{x}$ direction (columns) and the other estimating the gradient in the y-direction (rows). A convolution mask is usually much smaller than the actual image. As a result, the mask is slid over the image, manipulating a square of pixels at a time. The actual Sobel masks are shown below:

$$
\begin{aligned}
& \begin{array}{lll}
-1 & 0 & +1
\end{array} \\
& \mathrm{Gx}=\begin{array}{lll}
-2 & 0 & +2
\end{array} \\
& \begin{array}{lll}
-1 & 0 & +1
\end{array} \\
& +1+2+1 \\
& \mathrm{~Gy}=\begin{array}{lll}
0 & 0 & 0
\end{array} \\
& \begin{array}{lll}
-1 & -2 & -1
\end{array}
\end{aligned}
$$

The magnitude of the gradient is then calculated using the formula:

$$
|G|=\sqrt{G x^{2}+G y^{2}}
$$

An approximate magnitude can be calculated using:

$$
|G|=|G x|+|G y| \text {. }
$$

\subsubsection{Kernel Filter}

A kernel filter works by applying a kernel matrix to every pixel in the image. The kernel has multiplication factors to be used to the pixel and its neighbors. Once all the values have been multiplied, the pixel is changed with the sum of the products. By choosing different kernels, different types of filtering can be applied. Kernel filters provide low and high-pass filtering using a kernel.

Fig 1 : Sample Image and its Corresponding Histogram

\subsubsection{The Low-pass Filter}

3.2.4 A low pass filter is the basis for most smoothing methods. An image is smoothed by decreasing the disparity between pixel values by averaging nearby pixels. Using a low pass filter tends to retain the low frequency information within an image while reducing the high frequency information. An example is an array of ones divided by the number of elements within the kernel, such as the following 3 by 3 kernel:
$-1 / 16-1 / 8-1 / 16$
$-1 / 8 \quad 3 / 4 \quad-1 / 8$
$-1 / 16-1 / 8 \quad-1 / 16$

Proposed Hybrid KSL Filtering for MRI Noise removal method:

Input: Input Image

Output: Noise Reduced Image

Algorithm :

Step 1: Accept the input image

Step 2: Apply mask Gx,Gy to the input image

Step 3: Apply Kernel edge detection algorithm

Step 4: Masks manipulation of Gx,Gy separately on the input image

Step 5: Apply Sobel edge detection algorithm and the gradient

Step 6: Masks manipulation of Gx,Gy separately on the input image

Step 7: Apply Low-pass filter

Step 8: Masks manipulation of Gx,Gy separately on the input image

Step 9: Combine the filter Results to get a noise free output image.

\section{RESULTS \& DISCUSSION}

The proposed hybrid filtering KSL filtering algorithm is implemented in Matlab and tested with sample MRI clinical images and producing noteworthy results. Experimental results of this work can be discussed by seeing an example.

Step 1: Read the MRI image to reduce the noise (Fig 2).

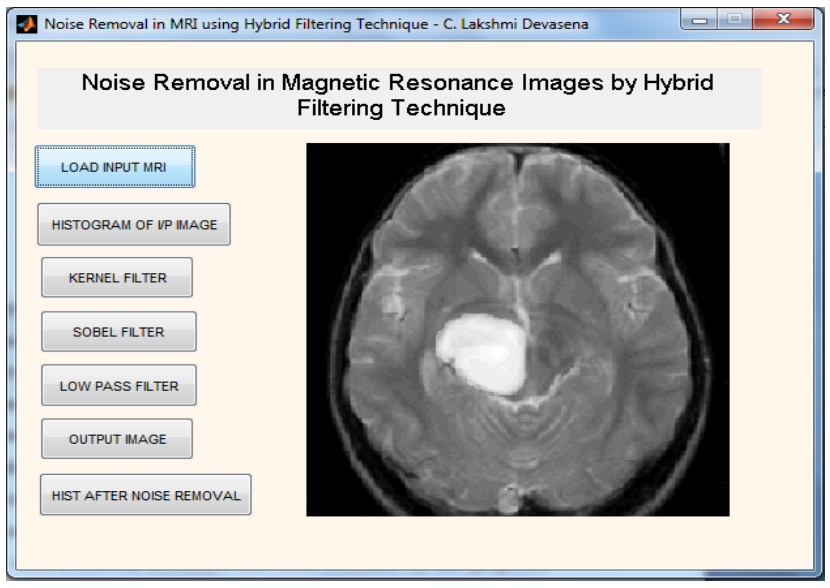

Fig 2: Load Input MRI Image 
Step 2: After reading the image, the existence of noise can be verified by seeing its histogram as shown in Fig 3.

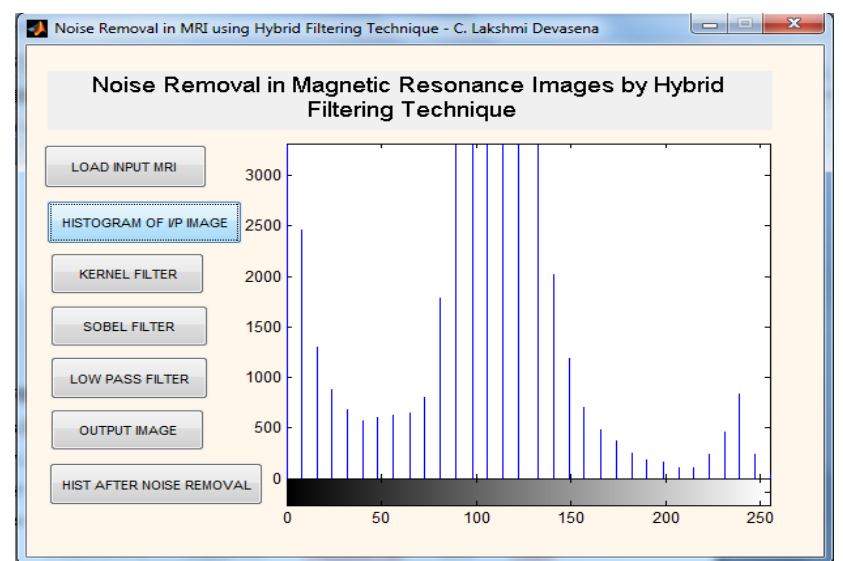

Fig 3: Histogram of the Input MR Image.

Step 3: Apply the kernel filter to the input image. The result of Kernel Filter is shown in Fig 4.

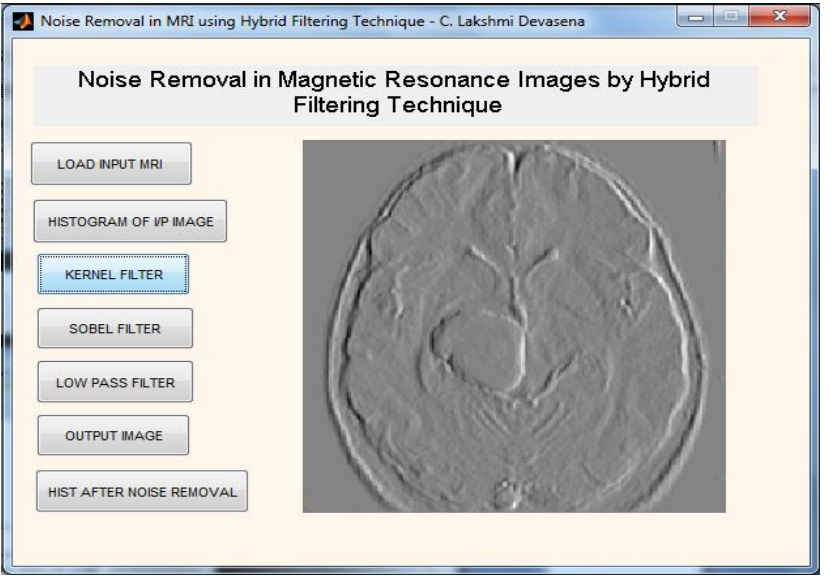

Fig 4: Result of Kernel Filter

Step 4: Apply the Sobel filter to the input image. The result of Sobel Filter is shown in Fig 5.

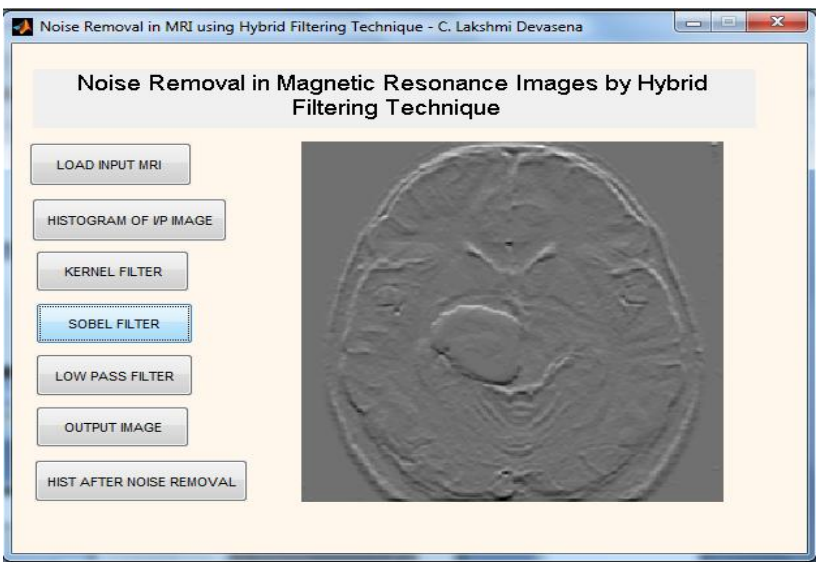

Fig 5: Result of Sobel Filter
Step 5: Apply the Low-pass filter to the input image. The result of Low-pass Filter is shown in Fig 6.

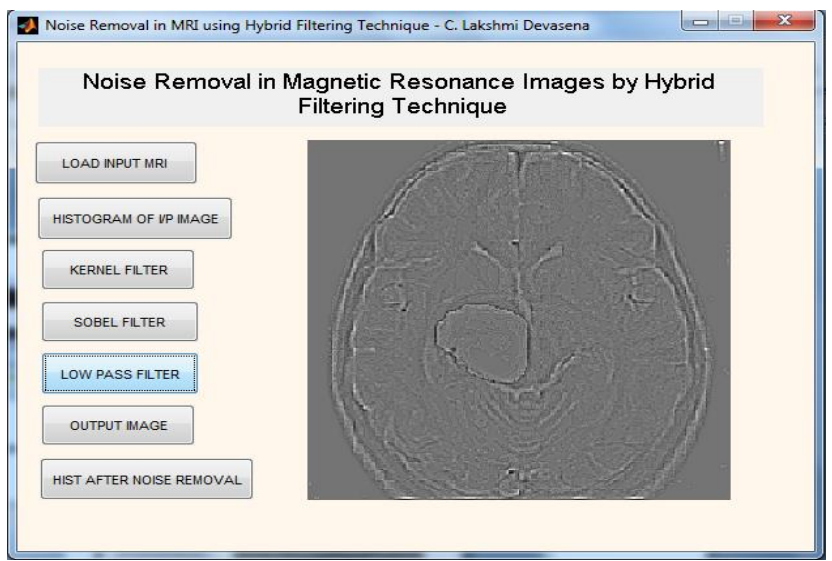

Fig 6: Result of Low-pass Filter

Step 6: Combine the filter results to get the noise free MR output image as shown in Fig 7.

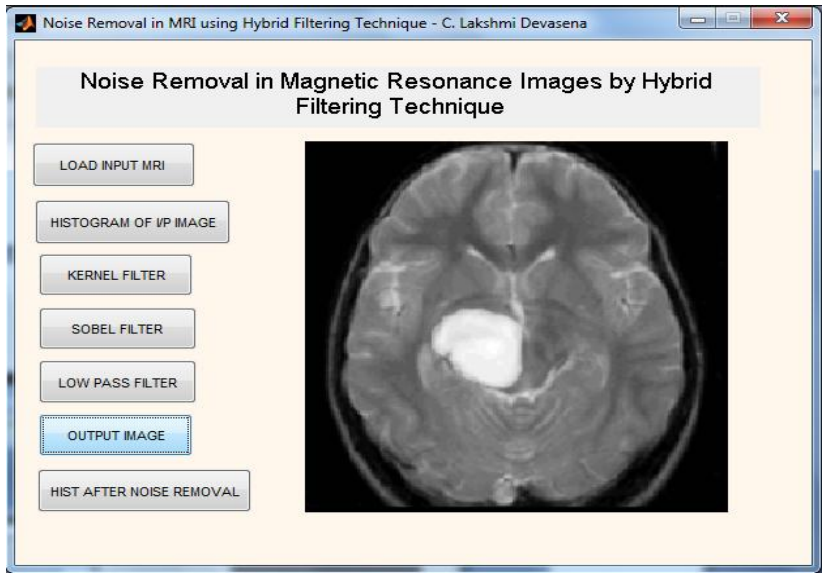

Fig 7: Noise Free output of the proposed method.

Step 7: Now we can verify the existence of noise in the produced output image by comparing its histogram. The histogram of the output image is shown in Fig 8. Now we can notify the difference between two histograms and ensure the removal of noise.

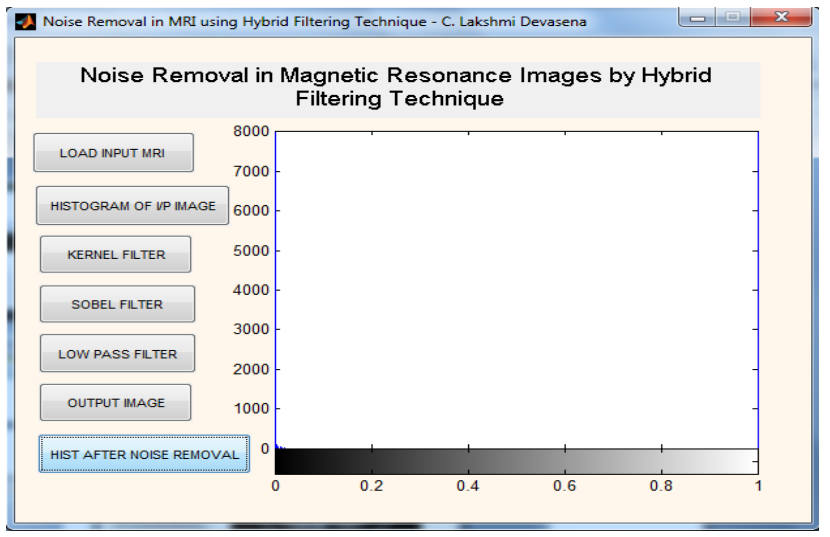

Fig 8: Histogram of the resulting image. 


\section{CONCLUSIONS}

The proposed hybrid KSL filtering technique for noise removal in Magnetic Resonance images is implemented in Matlab and tested with different synthetic and real clinical MRI images and producing promising results. This method could be used to reduce the noise in different type of MRI images like low-SNR MR images, partially parallel MRI images and so on. The output of this method has been compared with other filtering methods and shows superior performance.

\section{REFERENCES}

[1] Mukesh C. Motwani, Mukesh C. Gadiya, Rakhi C. Motwani and Frederick C. Harris "Survey of Image Denoising Techniques"

[2] J.Rajeesh, R.S.Moni, S.Palanikumar \& T.Gopalakrishnan "Noise Reduction in Magnetic Resonance Images using Wave Atom Shrinkage" International Journal of Image Processing (IJIP), Volume(4) : Issue(2) 2010 pp: 131-141.

[3] Milindkumar V. Sarode, Dr. Prashant R. Deshmukh "Performance Evaluation of Noise Reduction Algorithm in Magnetic Resonance Images" IJCSI International Journal of Computer Science Issues, Vol. 8, Issue 2, March 2011

[4] Milindkumar V. Sarode, Dr. Prashant R. Deshmukh "Performance Evaluation of Rician Noise Reduction Algorithm in Magnetic Resonance Images" Journal of Emerging Trends in Computing and Information Sciences Volume 2 Special Issue 2010-11 CIS Journal ISSN 2079 8407 pp 39-44.

[5] F. Kruggel, D. Y. von Cramon, and X. Descombes "Comparison of Filtering Methods for fMRI Datasets" NeuroImage 10, 530-543 (1999)

[6] Suyash P. Awate and Ross T. Whitaker "Nonparametric Neighborhood Statistics for MRI Denoising" Proc. Int. Conf. on Information Processing in Med. Imag., 2005.

[7] Lei Jiang,Wenhui Yang “Adaptive Magnetic Resonance Image Denoising Using Mixture Model and Wavelet Shrinkage" Proc. VIIth Digital Image Computing: Techniques and Applications, 10-12 Dec. 2003, Sydney.

[8] Yong Zhou and Jingfei Ma "Acoustic Noise Reduction in MRI by Selective Gradient Derating” Proc. Intl. Sot. Mag. Reson. Med. 82000.

[9] José V. Manjón, Neil A. Thacker, Juan J. Lull, Gracian Garcia-Martí, Luís Martí-Bonmatí, Montserrat Robles "Multicomponent MR Image Denoising" Hindawi Publishing Corporation International Journal of Biomedical Imaging Volume 2009, Article ID 756897, 10 pages doi: $10.1155 / 2009 / 756897$
[10] Jorge E. Pezoa "Noise Estimation and Removal for MRI Imaging” 2009.

[11] Miguel E. Soto, Jorge E. Pezoa and Sergio N. Torres" Thermal Noise Estimation and Removal in MRI: A Noise Cancellation Approach" 2011.

[12] G. Adluru, T. Tasdizen, R.T. Whitaker, and E. DiBella "Improving Undersampled MRI Reconstruction Using Non-Local Means" in Proc. ICPR, 2010, pp.4000-4003.

[13] Saurav Basu, Thomas Fletcher, and Ross Whitaker "Rician Noise Removal in Diffusion Tensor MRI” MICCAI 2006, LNCS 4190, pp. 117-125, 2006.

[14] M. Dylan Tisdall* and M. Stella Atkins "Using Human and Model Performance to Compare MRI Reconstructions" IEEE TRANSACTIONS ON MEDICAL IMAGING, VOL. 25, NO. 11, NOVEMBER 2006

[15] Guido Gerig, Olaf Kubler, Ron Kikinis, and Ferenc A. Jolesz "Nonlinear Anisotropic Filtering of MRI Data" IEEE Transactions on Medical Imaging, Volume 11 No. 2, June 1992.

[16] Weihong Guo and Feng Huang "Adaptive total variation based filtering for MRI images with spatially inhomogeneous noise and artifacts" IEEE 978-1-42443932-4/092009 pp: 101-104.

[17] W. Wong, A. Chung "Trilateral Filtering: A Non-linear Noise Reduction Technique for MRI” Proc. Intl. Soc. Mag. Reson. Med. 11 (2004)

[18] Wilbur C.K. Wong, Albert C.S. Chung "A nonlinear and non-iterative noise reduction technique for medical images: concept and methods comparison" International Congress Series 1268 (2004) 171- 176.

[19] Marius Lysaker, Arvid Lundervold, and Xue-Cheng Tai ” Noise Removal Using Fourth-Order Partial Differential Equation With Applications to Medical Magnetic Resonance Images in Space and Time" IEEE Transactions On Image Processing, VOL. 12, NO. 12, December 2003.

[20] Dylan Tisdall and M. Stella Atkins " MRI denoising via phase error estimation" Proc. of SPIE Vol. 5747 (SPIE, Bellingham, WA, 2005) 1605-7422/05/\$15 . doi: 10.1117/12.595677. pp: 646-654.

[21] Paul Bao and Lei Zhang "Noise Reduction for Magnetic Resonance Images via Adaptive Multiscale Products Thresholding", IEEE Transactions on Medical Imaging, Volume 22, No. 9, September 2003. pp: 1089-1099. 